\title{
Five-year change in visual acuity following cataract surgery in an older community: the Blue Mountains Eye Study
}

Department of

Ophthalmology

University of Sydney

Australia

Correspondence:

P Mitchell

Department of

Ophthalmology

(Centre for

Vision Research)

Westmead Hospital

University of Sydney

Hawkesbury Rd

Westmead, NSW 2145

Australia

Tel: +61 298457960

Fax: + 61298456117

E-mail: paul_mitchell@

wmi.usyd.edu.au

Received: 15 January 2003 Accepted in revised form: 6 May 2003

This study was supported by the Australian National Health and Medical Research Council (Grant 974159), Westmead Millennium and Save Sight Institutes, University of Sydney, and the Harvey and Mavis Thaw Medical Research Trust

The authors declare that they have no proprietary interest in this material
Abstract

Aims To assess the change in visual acuity following cataract surgery in the Blue Mountains Eye Study (BMES) population. Change in visual acuity was assessed by age, sex, baseline cataract type, and baseline visual acuity.

Methods A 5-year prospective follow-up of the population-based BMES cohort, who were initially examined in 1992. After 5 years, 2335 survivors of $3654 \mathbf{( 7 5 . 1 \% )}$ baseline BMES participants were re-examined. Slit-lamp and retro-illumination lens photographs were graded for the presence of incident cataract and evidence of cataract surgery. Visual acuity was measured using a $\log M A R$ chart, read at $2.4 \mathrm{~m}$. The main outcome measure was change in the number of logMAR letters correctly identified by eyes that underwent cataract surgery during the 5-year follow-up period. Results In a multiple linear regression model, age $(P<0.0001)$ and early age-related maculopathy $(\mathrm{ARM})$ at baseline $(P<0.0001)$ were found to affect adversely the postoperative visual acuity following the cataract surgery. As expected, eyes with any baseline cataract showed the greatest improvement in visual acuity after cataract surgery (right eyes: mean \pm s.e. change of $3.75 \pm 1.34$ letters; left eyes: mean change \pm s.e. of $6.7 \pm 0.99$ letters). There was also a statistically significant improvement in vision after cataract surgery in eyes with no significant lens opacity graded as present at baseline (right eyes: mean \pm s.e. change of $3.78 \pm 1.85$ letters; left eyes: mean change \pm s.e. of $2.68 \pm 1.33$ letters).

Conclusions Age and baseline cataract or ARM status, and baseline visual acuity were
J Panchapakesan, E Rochtchina and P Mitchell

determinants of the postoperative visual outcome in older persons who underwent cataract surgery in this community. Eye (2004) 18, 278-282. doi:10.1038/

sj.eye. 6700641

Keywords: cataract; cataract surgery; visual acuity; age-related maculopathy

\section{Introduction}

Cataract is the most common eye disease of elderly persons and is a major cause of visual impairment and blindness worldwide. ${ }^{1}$ In Western countries, cataract surgery is a most effective treatment, so that cataract surgery is now the most common surgical procedure among persons aged over 65 years. ${ }^{2}$

Modern trends for cataract surgery are to offer surgical treatment to eyes with better preoperative visual acuity than in the past. The upper age of patients considered suitable for cataract surgery has also increased in recent years. Blue Mountains Eye Study (BMES) data permit an assessment of the change in visual acuity among persons who underwent cataract surgery in the follow-up period, among those with and without baseline cataract.

\section{Materials and methods}

The BMES is a population-based survey of vision and common eye diseases in an urban Australian population aged 49 years or older. Participants were resident in two postcode areas in the Blue Mountains region, west of Sydney, Australia. The baseline survey methods and procedures have been previously described. ${ }^{3,4}$ The Study was approved by the Western Sydney Area Health Service Human Ethics 
Committee and signed informed consent was obtained from all participants. A detailed questionnaire was administered before participants underwent a comprehensive eye examination after pupil dilatation. Baseline examinations were performed during 19921994, when 3654 of the 4433 (82.4\%) eligible residents were examined.

The 5-year follow-up examinations were conducted during 1997-1999. Of the 3654 residents seen at baseline, 543 had died by the time of the 5-year follow-up examinations and 2335 of the survivors $(75.1 \%)$ were re-examined. The mean duration between examinations was 5.1 years. Of those not seen, $383(12.3 \%)$ had moved from the area and $394(12.7 \%)$ refused the examination. Those who returned for follow-up were younger than those who did not return (mean age 64.3 vs 65.6 years), were less likely to be female (57.2 vs $61.1 \%$ ), and were also less likely to have had previous cataract surgery (3.8 vs $6.5 \%$ ).

At both examinations, cataract was documented from both slit-lamp (Topcon SL-7e camera, Topcon Optical Co., Tokyo, Japan) and retroillumination (Neitz CT-R cataract camera, Neitz Instrument Co., Tokyo, Japan) lens photographs. Details of the photographic technique and grading $^{3,5}$ used in the BMES have been previously reported. The grading closely followed the Wisconsin Cataract Grading System, ${ }^{6}$ with good agreement found for assessments of both inter and intragrader reliability. $^{7}$

A history of past cataract surgery was taken and confirmed at both examinations as well as from the photographic grading. The presence and severity of nuclear, cortical, and posterior subcapsular (PSC) cataract was assessed for each eye. ${ }^{6}$ Nuclear cataract was defined on a five-level scale by comparison with a set of four standard slit-lamp photographs; for this study, level 4 or 5 was defined as nuclear cataract. The percentage area involved by cortical or PSC cataract in each eye was calculated from the estimated percentage area

involvement in each of nine segments of the lens divided by a grid. ${ }^{6}$

In the present study, cataract was considered to be present at baseline if the level of nuclear opacity was grade 4 or greater, if cortical opacity involved $\geq 5 \%$ of the total lens area or if any PSC opacity was present, as in the Beaver Dam Eye Study (BDES). ${ }^{8}$ The grading of incident age-related maculopathy (ARM) lesions closely followed methods used in the BDES, as previously described. ${ }^{3}$ Visual acuity was measured using a logMAR chart (Vectorvision CSV-1000 ${ }^{\mathrm{TM}}$ Vectorvision Inc., Dayton, $\mathrm{OH}$, USA), read at $2.4 \mathrm{~m}$. Subjects scoring less than 54 letters (6/7.5) were assessed using a Humphrey autorefractor. The autorefractor reading was then placed in a spectacle frame and a subjective refraction was performed according to the Beaver Dam modification of the ETDRS protocol.

Statistical Analysis System software (SAS Institute Inc., Cary, NC, USA) was used for tabulations and statistical analysis. Analysis of covariance was used to compare age-adjusted mean changes in visual acuity between the eyes that had or had not undergone cataract surgery. A multiple linear regression analysis was used to examine factors associated with the postoperative change in visual acuity.

\section{Results}

Of the 2335 persons examined at the 5-year follow-up, we excluded $117(5.0 \%)$ who had previous cataract surgery in one or both eyes. Of the remaining 2218 persons, 126 $(5.7 \%)$ underwent cataract surgery in their first or both eyes during the follow-up period. The incidence of cataract surgery increased from $0.3 \%$ (one case) among persons aged $49-54$ years at baseline to $17.4 \%$ (49 cases) among those aged 75 years or older, $P<0.001$. Cataract surgery was performed in both the eyes of 58 participants $(2.7 \%)$ and in the second eye of 24 participants $(43.1 \%)$, who were unilaterally phakic at baseline. Incident cataract surgery was slightly more frequent (6.0 vs 5.2\%) in women (77 cases) than in men (49 cases), with cataract surgical rates higher in women at all younger age groups other than for persons aged 75 years or older. Of all incident cataract surgical cases, $88.1 \%$ were performed on persons aged 65 years or older at baseline.

In right eyes that did not have cataract surgery over the period, the presence of significant baseline cortical and PSC cataract predicted the largest negative impact on visual acuity with an average 5-year loss of 35 $\log$ MAR letters. The mean vision reduction (in logMAR letters) was greater in nonoperated than in operated eyes, for those eyes with baseline cortical cataract only (4.36), baseline PSC only (4.67), or baseline nuclear cataract only (6.41). All left eyes with both cortical and PSC cataract had cataract surgery during the 5-year period.

The visual impact of cataract surgery is described in Table 1. The 5-year change in visual acuity in eyes with baseline cataract that underwent cataract surgery included a mean gain of $3.75 \log$ MAR letters in right eyes. For right eyes with no significant baseline cataract, a very similar mean gain of $3.78 \log$ MAR letters was observed.

After excluding cases with advanced diabetic retinopathy or late ARM, we performed multiple linear regression analyses to assess factors associated with 5 -year change in visual acuity, for right and left eyes separately (Table 2). Cataract surgery was the variable that was most strongly associated with improvement 
Table 1 A 5-year change in visual acuity (LogMAR letters) associated with incident cataract surgery

\begin{tabular}{|c|c|c|c|c|c|c|c|c|}
\hline \multirow[t]{2}{*}{ Eye } & \multirow{2}{*}{$\begin{array}{c}\text { Prevalent cataract } \\
\text { at baseline }\end{array}$} & \multirow{2}{*}{$\begin{array}{c}\text { Incident cataract } \\
\text { surgery }\end{array}$} & \multirow[t]{2}{*}{$\mathrm{n}$} & \multicolumn{2}{|c|}{ Crude change } & \multicolumn{2}{|c|}{ Age-adjusted change } & \multirow[t]{2}{*}{$\mathrm{P}$} \\
\hline & & & & Mean & s.d. & Mean & s.e. & \\
\hline \multirow[t]{4}{*}{ Right } & No & No & 1203 & -2.56 & 7.89 & -3.11 & 0.27 & \\
\hline & No & Yes & 25 & 1.72 & 9.07 & 3.78 & 1.85 & 0.0003 \\
\hline & Yes & No & 268 & -6.16 & 10.35 & -4.32 & 0.59 & \\
\hline & Yes & Yes & 49 & 1.33 & 25.94 & 3.75 & 1.34 & 0.0001 \\
\hline \multirow[t]{4}{*}{ Left } & No & No & 1215 & -2.61 & 5.49 & -2.92 & 0.21 & \\
\hline & No & Yes & 30 & 1.67 & 15.59 & 2.68 & 1.33 & 0.0001 \\
\hline & Yes & No & 243 & -5.94 & 8.13 & -4.84 & 0.48 & \\
\hline & Yes & Yes & 56 & 5.14 & 20.85 & 6.7 & 0.99 & 0.0001 \\
\hline
\end{tabular}

Table 2 Factors associated with 5-year change in visual acuity in a multiple linear regression model

\begin{tabular}{llccl}
\hline Eye & Variable & $\begin{array}{c}\text { Parameter } \\
\text { estimate }\end{array}$ & $\begin{array}{c}\text { Standard } \\
\text { error }\end{array}$ & P-value \\
\hline Right & & & \\
& Intercept & 11.67 & 1.97 & 0.0001 \\
& Age (per year) & -0.23 & 0.03 & 0.0001 \\
& Sex & 0.22 & 0.45 & 0.62 \\
Early ARM & -9.1 & 1.27 & 0.0001 \\
& Any cataract & -1.36 & 0.61 & 0.03 \\
& Incident cataract Surgery & 6.06 & 1.09 & 0.0001 \\
& & & \\
Left & & & \\
& Intercept & 8.8 & 1.57 & 0.0001 \\
& Age (per year) & -0.18 & 0.02 & 0.0001 \\
& Sex & 0.02 & 0.36 & 0.95 \\
Early ARM & -4.44 & 1 & 0.0001 \\
Any cataract & -1.41 & 0.5 & 0.005 \\
Incident cataract Surgery & 8.58 & 0.81 & 0.0001 \\
\hline
\end{tabular}

ARM=age-related maculopathy.

in visual acuity. Conversely, age and presence of early $\mathrm{ARM}$ at baseline were more strongly associated with the 5-year loss of visual acuity. Early ARM was associated with a much larger 5-year visual acuity loss (-9.1 logMAR letters in right eyes; $-4.4 \log$ MAR letters in left eyes) than the 5-year visual acuity loss associated with the presence of any cataract at baseline (-1.4 logMAR letters in right eyes; $-1.4 \log$ MAR letters in left eyes). The multiple linear regression model indicated an average gain associated with cataract surgery of $6.06 \log$ MAR letters in right eyes and 8.58 letters in left eyes.

After adjusting for sex, the presence of early ARM or cataract at baseline, and incident cataract surgery, age was found to be a significant $(P<0.0001)$ predictor of fall in visual acuity over the 5-year period. For each decade of age, there was an adjusted loss of $2.3 \log$ MAR letters in right eyes and $1.8 \log$ MAR letters in left eyes.

Table 3 demonstrates the relation between baseline and follow-up visual acuity levels. A total of $46.7 \%$ of eyes undergoing cataract surgery during the follow-up period had better vision than at baseline, while $39.9 \%$ had similar visual acuity at follow-up and $13.4 \%$ of eyes had worse vision at follow-up. Of the 39 cases with baseline visual acuity $>6 / 6$, a total of $11(28.3 \%)$ had worse visual acuity at follow-up due to any cause. Follow-up visual acuity was $\geq 6 / 12$ in $90.1 \%$ of eyes. The proportion of eyes with worse follow-up visual acuity than at baseline decreased to $0 \%$ for cases with baseline visual acuity $\leq 6 / 60$.

\section{Discussion}

The mean gain in visual acuity from cataract surgery during the 5-year follow-up period was relatively modest. After accounting for the effects of age and presence of early ARM, the mean gain of $6.06 \log$ MAR letters in right eyes and $8.58 \log$ MAR letters in left eyes converts to a gain of around only one line of Snellen acuity.

There are a number of possible reasons that could explain this finding. First, eyes that had undergone cataract surgery in the period just prior to their attendance in the follow-up study are likely to have had worse visual acuities than recorded at their baseline examinations. Our study found that persons with baseline cataract would have lost a further mean 1.36 logMAR letters (in right eyes) and $1.41 \log$ MAR letters (in left eyes), prior to undergoing surgery during the 5-year period.

Second, PSC is a faster growing cataract type that becomes more visually disabling earlier than other cataract types, particularly for reading vision. It is possible many eyes without cataract at baseline may have developed this type of opacity during the follow-up period, discounting the overall 5-year visual acuity gain from the cataract procedure.

Finally, $\log$ MAR visual acuity measures only high contrast, monochrome distance vision under controlled 
Table 3 Visual acuity level following cataract surgery by baseline visual acuity level $(\%(n))$

\begin{tabular}{|c|c|c|c|c|c|c|c|}
\hline & \multicolumn{6}{|c|}{ Visual acuity level at 5 years in eyes having incident cataract surgery } & \multirow{2}{*}{$\begin{array}{c}\text { Follow-up } \\
\text { visual acuity } \\
<\text { baseline visual } \\
\text { acuity (\%) }\end{array}$} \\
\hline & $>6 / 6$ & $6 / 6-6 / 9$ & $6 / 9-6 / 12$ & $6 / 12-6 / 18$ & $6 / 18-6 / 60$ & $\leq 6 / 60$ & \\
\hline \multicolumn{8}{|c|}{ Baseline vision } \\
\hline$>6 / 6$ & $71.8(28)$ & $23.1(9)$ & $0(0)$ & $2.6(1)$ & $2.6(1)$ & $0(0)$ & 28.3 \\
\hline $6 / 6-6 / 9$ & $33.7(34)$ & $46.5(47)$ & $6.9(7)$ & $5.0(5)$ & $5.0(5)$ & $5.0(5)$ & 19.9 \\
\hline $6 / 9-6 / 12$ & $20.0(5)$ & $60.0(15)$ & $4.0(1)$ & $4.0(1)$ & $4.0(1)$ & $8.0(2)$ & 16 \\
\hline $6 / 12-6 / 18$ & $21.9(7)$ & 53.1 (17) & $12.5(4)$ & $12.5(4)$ & $0(0)$ & $0(0)$ & 0 \\
\hline $6 / 18-6 / 60$ & $12.5(1)$ & $62.5(5)$ & $0(0)$ & $12.5(4)$ & $0(0)$ & $12.5(1)$ & 12.5 \\
\hline$\leq 6 / 60$ & $40.0(2)$ & $20.0(1)$ & $0(0)$ & $0(0)$ & $20.0(1)$ & $20.0(1)$ & 0 \\
\hline
\end{tabular}

mesopic, low-glare lighting conditions. It thus represents only a fraction of the visual tasks required during an average day. Cataract surgery could have resulted in a more significant improvement in glare reduction, contrast sensitivity and reading or night vision than indicated by the recorded changes in $\log$ MAR acuity. Attempts have been made to describe visual function tests that may be more complete indicators of change in vision than $\log$ MAR or more commonly used Snellen acuity levels. ${ }^{21}$ Future studies including such visual function tests may provide a more global measure of visual outcomes after cataract surgery.

In our multivariate model of factors predicting 5-year change in visual acuity, increasing age was associated with a significant, though relatively modest loss of bestcorrected visual acuity. This represents the gradual deterioration of the visual system with age even when obvious pathology was not evident or could be adjusted for. It may be questioned whether there is a visual acuity cutoff for each age beyond which cataract surgery may provide limited benefit. This is difficult to answer as the impact of modest vision loss varies between individuals. For example, impaired vision from cataract (reducing visual acuity to below 6/12, the level required to maintain a drivers licence) may cause a greater impact on older persons who are still driving than on those who do not drive.

The BDES, on whose methodology our study was modelled, also found that cataract surgery had less positive impact on visual acuity than might have been expected. The mean number of logMAR letters gained, after adjustment, following cataract surgery during a 5-year interval was quite similar to that in our study. In the BDES report, ${ }^{9}$ there was a greater gain in right than in left eyes, whereas in our study, the gain in left eyes was greater than in right eyes.

Although our study found that better levels of baseline visual acuity were associated with a greater likelihood of worse vision after cataract surgery at the follow-up examination, we are not aware of the vision immediately before surgery. It is possible that rapidly developing cataract type (eg PSC) could have had a significant effect on vision between the time of the baseline examination and the time of surgery.

The mean visual acuity improvement following cataract surgery in our study compares reasonably with that reported by other centres. A Singapore study ${ }^{10}$ found that $85.1 \%$ of patients reported improved acuity and $77.6 \%$ reported better visual function after cataract surgery. An Auckland study ${ }^{11}$ of 488 eyes found that postcataract surgery visual acuity was $6 / 12$ or better in $88 \%$ of eyes. A Hong Kong ${ }^{12}$ study reported that $59.6 \%$ of eyes had a visual acuity of $6 / 18$ or better after surgery. A Moorfields Eye Hospital report of 1676 eyes $^{13}$ found that at discharge, $95.4 \%$ of eyes without any preoperative morbidity achieved a visual acuity of $6 / 12$ or better.

A Swedish study ${ }^{14}$ used a self-assessed visual function questionnaire to examine pre- and postoperative satisfaction with first compared with second eye cataract surgery. A clinical index ${ }^{15}$ for predicting potential improvement used age, preoperative acuity, reading frequency, together with comorbidities was found to predict postoperative acuity within one Snellen line in $72 \%$ of cases. Visual function assessments or clinical indices may permit more global assessments of the benefits from cataract surgery than simply change in acuity. At this point, however, no single broad measure of visual function is accepted.

Setty et $a l^{16}$ examined the changing thresholds for cataract surgery, while age effects were studied by Westcott et al. ${ }^{17}$ The latter report examined 880 patients aged over 60 years who had cataract surgery. In patients with no comorbidities, the odds of achieving 6/12 or better acuity was 4.6 times higher among persons aged 60-69 years than among persons aged 80 years or older.

In Australia, a large component of the cost of cataract surgery is borne by government agencies and leads to a need for predictions of future cataract demands. Taylor 
and co-workers ${ }^{18}$ have used data from the Melbourne Visual Impairment Project to generate estimates of the cataract surgery burden in Australia.

In Western communities, the relatively high prevalence of ARM lesions in older patients being considered for cataract surgery makes even moderate changes in outcome, significant in population terms. Our study demonstrated a major negative impact from the presence of early ARM lesions on 5-year visual outcomes after cataract surgery. Although Shuttleworth $e t a l^{19}$ also questioned whether patients with ARM benefited from cataract surgery, $67 \%$ of patients in this study still felt that the operation was worthwhile. While earlier cataract surgery could be considered in order to optimise the potential for visual improvement in patients with early or moderate ARM, there remain concerns regarding the potential risk from progression of these early-stage ARM lesions, associated with surgery. Recent pooled 5-year data from two older population-based cohorts (the BDES and our own study) showed a five-fold risk of incident late-stage ARM in eyes that previously had cataract surgery, after adjusting for ARM risk factors and the presence of early-stage ARM lesions. ${ }^{20}$

An important limitation of our study is that its design, a fixed 5-year follow-up period, with no routine prospective collection of data on the level of visual acuity just prior to cataract surgery or on the type of cataract present at that time, reduces our ability to explore shorter-term changes in vision following cataract surgery.

In summary, our data indicate that cataract surgery is associated with an improvement in visual acuity over the intermediate term in the majority of cases. The average visual acuity improvement documented in this population appears to be relatively modest, which is in keeping with data from another population-based study and from other clinical studies. The decision to recommend surgery will always need to take individual parameters into account. Clinical guidelines for cataract surgery are relatively blunt instruments. Their widespread application could result in many patients being denied access to surgery that could provide substantial benefit to quality of life and independent living.

\section{References}

1 Hyman L. Epidemiology of eye disease in the elderly. Eye 1987; 1: 330-341.

2 Steinberg EP, Javitt JC, Sharkey PD, Zuckerman A, Legro MW, Anderson GF et al. The content and cost of cataract surgery. Arch Ophthalmol 1993; 111: 1041-1049.
3 Mitchell P, Smith W, Attebo K, Wang JJ. Prevalence of agerelated maculopathy in Australia. The Blue Mountains Eye Study. Ophthalmology 1995; 102: 1450-1460.

4 Attebo K, Mitchell P, Smith W. Visual acuity and the causes of visual loss in Australia. The Blue Mountains Eye Study. Ophthalmology 1996; 103: 357-364.

5 Mitchell P, Cumming RG, Attebo K, Panchapakesan J. Prevalence of cataract in Australia: the Blue Mountains eye study. Ophthalmology 1997; 104: 581-588.

6 Klein BE, Klein R, Linton KL, Magli YL, Neider MW. Assessment of cataracts from photographs in the Beaver Dam Eye Study. Ophthalmology 1990; 97: 1428-1433.

7 Panchapakesan J, Cumming RG, Mitchell P. Reproducibility of the Wisconsin cataract grading system in the Blue Mountains Eye Study. Ophthalmic Epidemiol 1997; 4: 119-126.

8 Klein BE, Klein R, Linton KL. Prevalence of age-related lens opacities in a population. The Beaver Dam Eye Study. Ophthalmology 1992; 99: 546-552.

9 Klein BE, Klein R, Moss SE. Change in visual acuity associated with cataract surgery. The Beaver Dam Eye Study. Ophthalmology 1996; 103: 1727-1731.

10 Saw SM, Tseng P, Chan WK, Chan TK, Ong SG, Tan D. Visual function and outcomes after cataract surgery in a Singapore population. J Cataract Refract Surg 2002; 28: 445-453.

11 Riley AF, Malik TY, Grupcheva CN, Fisk MJ, Craig JP, McGhee CN. The Auckland cataract study: co-morbidity, surgical techniques, and clinical outcomes in a public hospital service. Br J Ophthalmol 2002; 86: 185-190.

12 Lau J, Michon JJ, Chan WS, Ellwein LB. Visual acuity and quality of life outcomes in cataract surgery patients in Hong Kong. Br J Ophthalmol 2002; 86: 12-17.

13 Murphy C, Tuft SJ, Minassian DC. Refractive error and visual outcome after cataract extraction. J Cataract Refract Surg 2002; 28: 62-66.

14 Lundstrom M, Brege KG, Floren I, Stenevi U, Thorburn W. Impaired visual function after cataract surgery assessed using the Catquest questionnaire. J Cataract Refract Surg 2000; 26: 101-108.

15 Graney MJ, Applegate WB, Miller ST, Elam JT, Freeman JM, Wood TO et al. A clinical index for predicting visual acuity after cataract surgery. Am J Ophthalmol 1988; 105: 460-465.

16 Setty R, Bosanquet R, Harle J. Changing thresholds for cataract surgery. Br J Ophthalmol 2000; 84: 1439.

17 Westcott MC, Tuft SJ, Minassian DC. Effect of age on visual outcome following cataract extraction. Br J Ophthalmol 2000; 84: 1380-1382.

18 McCarty CA, Keeffe JE, Taylor HR. The need for cataract surgery: projections based on lens opacity, visual acuity, and personal concern. Br J Ophthalmol 1999; 83: 62-65.

19 Shuttleworth GN, Luhishi EA, Harrad RA. Do patients with age related maculopathy and cataract benefit from cataract surgery? Br J Ophthalmol 1998; 82: 611-616.

20 Wang JJ, Klein R, Smith W, Klein BEK, Tomany S, Mitchell P. Cataract surgery and the 5-year incidence of late-stage agerelated maculopathy: pooled findings from the Beaver Dam and Blue Mountains Eye Studies. Ophthalmology 2003; 110: 1960-1967.

21 Ivers RQ, Optom B, Macaskill P, Cumming RG, Mitchell P. Sensitivity and specificity of tests to detect eye disease in an older population. Ophthalmology 2001; 108: 968-975. 